Polymer Journa1, Vol. 8, No. 2, pp 202-207, (1976)

\title{
Complex Formation of Poly-L-lysine with Poly(acrylic acid)
}

\author{
Katsuro Shinoda, Toshio Hayashi, Toru Yoshida, \\ Kazuhiko SAKaI, and Akio NAKajima* \\ Department of Polymer Chemistry, Kyoto University, \\ Sakyo-ku, Kyoto 606, Japan.
}

(Received Oct. 28, 1975)

\begin{abstract}
Conformation-directing interactions between poly-L-lysine (PLL) and poly(acrylic acid) (PAA) in aqueous media have been studied as a function of $\mathrm{pH}$ by circular dichroism spectroscopy. It was found that PAA reacts with PLL stoichiometrically independent of the molecular weight of the PAA and induces $\alpha$-helix formation for PLL. The induced circular dichroism spectra of the complex have been measured by using acridine orange as a chromophore. The results, together with the $\mathrm{CD}$ spectra in the ultraviolet region, suggest that PAA which is capable of forming a left-handed super helix binds to the core composed of a right-handed $\alpha$-helix of PLL.

KEY WORDS Polyelectrolyte Complex / Poly-L-lysine / Poly(acrylic acid) / Conformations / Circular Dichroism / Induced Circular Dichroism /
\end{abstract}

The interactions between poly-L-lysine (PLL) and acidic polyelectrolyte have been widely investigated as a model for a variety of biological systems, such as nucleoproteins and proteinpolysaccharide complexes. Various kinds of polyacids of either synthetic or natural origin have been used as the partner polyelectrolytes, e.g., DNA, RNA, poly(adenylic acid), phosvitin, and mucopolysaccharides. Several investigators ${ }^{1-3}$ suggested that these interactions were principally ionic in nature but that they would be dependent on the geometry and structure of the component polymers as well as the number of ionic groups.

We have been interested in the effect of the conformation and the configuration of the component polymers on the interactions. ${ }^{4}$

Poly(acrylic acid) (PAA) would be one of the most adequate acidic components to elucidate the effect of the chemical structure on the complex formation with PLL, because of the flexibility of the backbone chain and the structural simplicity. However, very little has been reported $^{5,6}$ about the interaction of PAA with PLL. Gratzer and $\mathrm{McPhie}^{5}$ suggested that the complexes existed in $\alpha$-helices at neutral $\mathrm{pH}$, where PLL was in the "charged coill" form in

* To whom correspondence should be addressed. the absence of PAA, and that the helical content of the PLL was invariably less than about $50 \%$ in aqueous solution. On the other hand, Zezin, et al. ${ }^{6}$ suggested that the helical content of PLL in the PLL-PAA complex was about $70 \%$ at $\mathrm{pH} 4$ in $\mathrm{H}_{2} \mathrm{O}$-ethanol solution.

The purpose of the present study is to elucidate in detail the interaction in aqueous solutions as a function of both the $\mathrm{pH}$ and the PLL/PAA ratio and to examine the structure of the complex by using circular dichroism spectroscopy.

\section{EXPERIMENTAL}

\section{Materials}

Poly-L-lysine (PLL) was prepared by decarbobenzyloxylation from poly( $\varepsilon$-carbobenzyloxy-Llysine) (PCBLL) that was synthesized by the NCA method, according to the procedures previously described. ${ }^{4}$ The molcular weight of PLL estimated from the osmotic pressure measurements on PCBLL and from the limiting viscosity number $[\eta]$ of PLL was about 238,000.

Poly(acrylic acid)-Na samples of different molecular weights, designated by PAA-H and PAA-L, were the products of Toa Gosei Chemical, Co., Ltd. and were used after precipitations with ethanol. The molecular weights of the samples calculated from $[\eta]$ in $1.5 \mathrm{~mol} / l \mathrm{NaBr}$ 
aqueous solution at $15^{\circ} \mathrm{C}$ were $2.9 \times 10^{5}$ (for PAA-H) and $7.5 \times 10^{3}$ (for PAA-L).

Acridine orange (AO) (CHROMA) was purified by the method reported by Myhr and Foss. ${ }^{7}$

\section{Preparation of Mixtures}

Separate dilute aqueous solutions were prepared for PLL and PAA. The PLL solution was adjusted at $\mathrm{pH} 12$ by adding $1.0-\mathrm{N} \mathrm{NaOH}$. Mixtures were prepared by slow dropwise addition of an aqueous solution of PAA at $\mathrm{pH} c a .7$ to the PLL solution at $\mathrm{pH} \mathrm{12;} \mathrm{then} \mathrm{the} \mathrm{pH}$ of the mixture was brought to the desired values by adding $\mathrm{HCl}$, and neutralized $0.01-N$ AO aqueous solution was added if necessary. The relative proportions of the two polymer components were quoted as the residue-mole ratio. Some mixtures showed slight turbidity, especially in the vicinity of the stoichiometric composition at the given $\mathrm{pH}$. However, the $\mathrm{CD}$ spectrum seems to be affected by the turbidity hardly at all (see the following paper $^{8}$ ).

\section{Circular Dichroism}

The circular dichroism (CD) spectra were measured at $25 \pm 0.5^{\circ} \mathrm{C}$ using a JASCO J-20 CD/ORD Spectropolarimeter equipped with a quartz cell of path length $1 \mathrm{~mm}$. The PLL concentration in mixtures was from 0.002 to $0.003 \mathrm{M}$. The concentrations were determined by conductometric titration and/or gravimetry. The residue ellipticity, $[\theta]$ in degree $\cdot \mathrm{cm}^{2} \cdot \mathrm{dmol}^{-1}$, for the mixtures was calculated based on the mole concentration of the PLL, except in the system including $\mathrm{AO}$, for which $[\theta]$ was calculated based on the mole concentration of AO.

\section{pH Measurements}

The $\mathrm{pH}$ values of the solutions were measured with a Hitachi-Horiba $\mathrm{pH}$-Meter Model F-7 equipped with a combination $\mathrm{pH}$ electrode 6028-10T, the sensitivity of which was $0.005 \mathrm{pH}$. $\mathrm{pH}$ titrations were performed in the presence of $0.02 \mathrm{M} \mathrm{NaCl}$ under nitrogen atomasphere in order to determine the state of charge of the PLL and the PAA under the condition of the CD measurements.

\section{RESULTS AND DISCUSSION}

\section{Spectra of PLL-PAA Mixtures}

When PLL reacts with PAA in aqueous solu- tion, a polyelectrolyte complex is formed. The structure of the complex would mainly depend on the $\mathrm{pH}$ of the solution, because both components are weak polyelectrolytes. The CD spectra of the $47: 53$ and the $68: 32$ mole ratio PLL-PAA-H mixtures at various $\mathrm{pH}$ values are shown in Figures 1 and 2, respectively. These

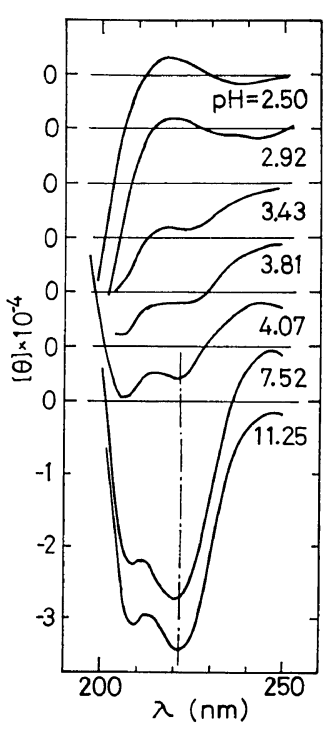

Figure 1. CD spectra of $47: 53$ mole ratio PLLPAA-H mixture at $25^{\circ} \mathrm{C}$ and various $\mathrm{pH}$ values. Broken line indicates the position of $222 \mathrm{~nm}$.

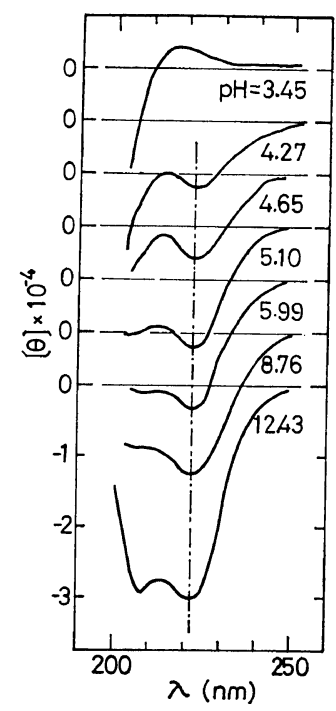

Figure 2. CD spectra of $68: 32$ mole ratio PLLPAA-H mixture at $25^{\circ} \mathrm{C}$ and various $\mathrm{pH}$ values. 
spectra give information about the conformation of the PLL in the complex, because PAA has no strong absorption band in this wavelength region. Comparison of Figures 1 and 2 with the spectra of PLL in aqueous solution (Figure 3) suggests that the conformation of the PLL in the complex is based on the conformations of the $\alpha$-helix and of the charged coil. This is because the characteristic bands ${ }^{9}$ of the $\alpha$-helix (a negative $222 \mathrm{~nm}$ band assigned to the $\mathrm{n}-\pi^{*}$

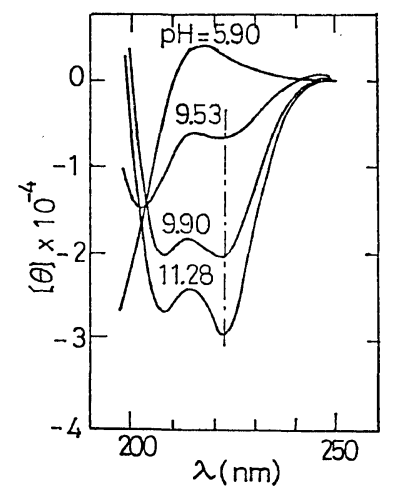

Figure 3. CD spectra of PLL in aqueous solution at $25^{\circ} \mathrm{C}$ and various $\mathrm{pH}$ values.

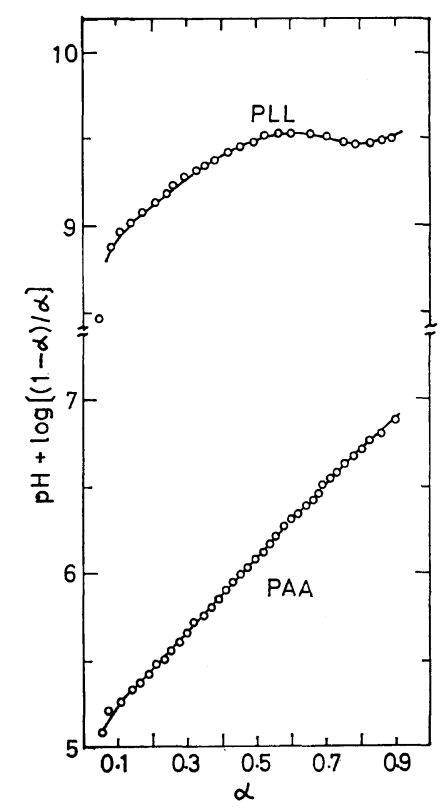

Figure 4. Potentiometric titration curves of PLL and PAA at $25^{\circ} \mathrm{C}$ : Polymer concn, $0.0025 \mathrm{~N} ; \mathrm{NaCl}$ concn, $0.02 \mathrm{~N}$. transition and a negative $208 \mathrm{~nm}$ band assigned to the $\pi-\pi^{*}$ transition) and of the charged coil (a positive $218 \mathrm{~nm}$ band) are unchanged for the PLL-PAA-H mixtures. However, the PLL adopts the $\alpha$-helical conformation in the neutral $\mathrm{pH}$ region rather than the charged coil observed in the absence of PAA.

Figure 4 shows the titration curves for $0.0025 \mathrm{~N}$ PLL and $0.0025 N$ PAA in $0.02 N \mathrm{NaCl}$, represented by the degree of dissociation $\alpha$ (for the reactions $\mathrm{RNH}_{3}{ }^{+}=\mathrm{RNH}_{2}+\mathrm{H}^{+}$and $\mathrm{RCOOH}=$ $\mathrm{RCOO}^{-}+\mathrm{H}^{+}$, respectively, for the $\varepsilon$-amino group of PLL and the carboxyl group of PAA) vs. $\mathrm{pH}+\log (1-\alpha) / \alpha$. For PAA only one curve is shown in the figure, because the plots were independent of the molecular weights. ${ }^{10}$

In the neutral $\mathrm{pH}$ region, both carboxyl and $\varepsilon$-amino groups are almost fully ionized. Therefore, the stabilization of the helical structure of PLL may be caused by the neutralization of the $\varepsilon$-amino group in the side chain of PLL by the carboxyl group of PAA.

With respect to one spectrum ( $\mathrm{pH} 7.52$ in Figure 1), the $222 \mathrm{~nm}$ band seems to shift to $220 \mathrm{~nm}$. This may suggest the existence of a $\beta$-structure to some extent. The $\beta$-structure of PLL was characterized by a negative $217 \mathrm{~nm}$ band $([\theta]=$ $-19,300)$ and a positive $195 \mathrm{~nm}$ band $([\theta]=$ $+28,000)$. However, in this case, the numerical value of $[\theta]$ at $222 \mathrm{~nm}([\theta]=-33,000)$ was equal to that for a typical $\alpha$-helix. This fact may indicate that the proportion of $\beta$-structure of PLL in the complex is small.

\section{Conformation Change of $P L L$ in $P L L-P A A$ Mixtures as a Function of $p H$}

In Figure 5, the residue ellipticity at $222 \mathrm{~nm}$, $-[\theta]_{222}$, is plotted against $\mathrm{pH}$ for mixtures containing different ratios of PLL and PAA-H; here data not shown in previous figures are also included. PLL shows a sharp transition at about $\mathrm{pH}$ 9.5, but the helix region is broadened by the existence of the PAA. When the content of PLL is larger than that of PAA-H, the curves give a two-step transition (see curves 1 and 2 in Figure 5). With the molar ratio of PLL/ PAA-H less than 1, however, a sharp change of $-[\theta]_{222}$ occurs in between $\mathrm{pH} 3$ and 6 , thereafter the value remains essentially constant. As mentioned above, such stabilization 


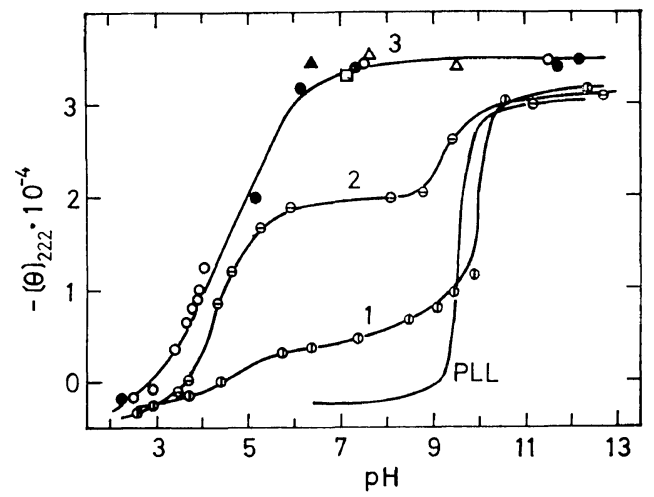

Figure 5. $-[\theta]_{222}$ plotted against $\mathrm{pH}$ for PLLPAA-H mixtures and for PLL in aqueous solutions. The mole ratios of PLL to PAA-H are: (1) (1) $86: 14$; (2) $\ominus 68: 32$; and (3) $\square 50: 50 ; \bigcirc, 47: 53$; $\triangle, 34: 66 ; \Delta, 25: 75 ; \bigcirc, 19: 81$.

of the helical structure of PLL by PAA would be caused by the neutralization of $\varepsilon$-amino groups by carboxyl groups. In order to examine the electrochemical stoichiometry of this reaction, a plot of ellipticity, $-[\theta]_{222}$, at $\mathrm{pH} 7.5$ is illustrated as a function of PAA-H mole fraction in Figure 6. Both PLL and PAA are almost ionized at this $\mathrm{pH}$. The fact that an approximately linear relationship exists between the $-[\theta]_{222}$ and PAA-H mole fraction shows that these interactions are performed stoichiometrically. Furthermore, it is obvious, from the titration curves, that the first step transition (at lower $\mathrm{pH}$ ) and the second one (at higher $\mathrm{pH}$ ) of curves 1 and 2 in Figure 5 correspond to the dissociation of carboxyl and $\varepsilon$-amino groups,

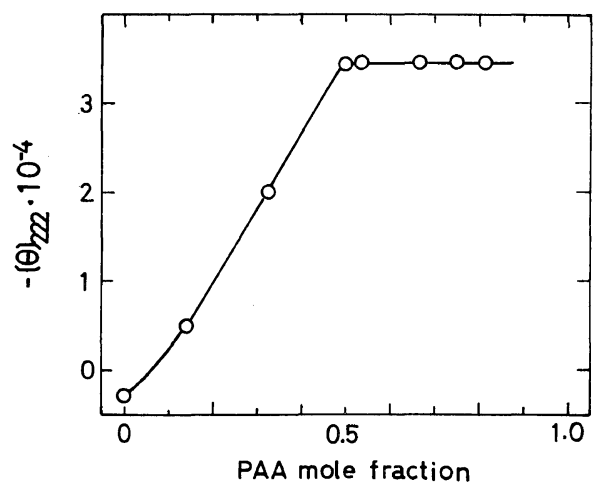

Figure 6. $-[\theta]_{222}$ plotted against $\mathrm{PAA}-\mathrm{H}$ mole fraction in the PLL-PAA-H mixtures at $\mathrm{pH}$ 7.5. respectively. Naturally, in the case when the content of PLL is smaller than that of PAA-H, only the first step transition would occur, because all the PLL would exist in the $\alpha$-helix even at neutral $\mathrm{pH}$.

A helix-to-coil transition of PLL takes place at about the degree of dissociation $\alpha$ equal to 0.65 (35\% charged) (see Figure 4). If we assume that the helix-to-coil transition of PLL in the PLL-PAA complex takes place at the same $\alpha$ as for PLL by itself, the transition will occur in the $\mathrm{PLL} / \mathrm{PAA}=1.0$ system at the $\mathrm{pH}$ value at which PAA has the $\alpha$ value of 0.65 . Such an estimated $\mathrm{pH}$ value is about 6.5 from the titration curve in Figure 4. However, the experimental value of the midpoint of transition of curve 3 in Figure 5 is about 4.5. This difference may be caused by some induced dissociation of carboxyl groups with the coexisting $\varepsilon$-amino groups of PLL.

One of the characteristic features of curve 3 in Figure 5 is that the numerical values of $-[\theta]_{222}$ of the mixtures at the helical region are larger than that of PLL. The reported values of $[\theta]_{222}$ for $\alpha$-helix of PLL in aqueous solution were $-35,000$ by Tiffany, et al., ${ }^{12}-30,000$ by Hatano, et al., ${ }^{13}$ and $-28,000$ by Gelman, et al., ${ }^{14}$ all which are considerably smaller than the value $-40,000$ reported for the perfect $\alpha$ helix of poly(L-glutamic acid). It is not known whether these differences in $[\theta]_{222}$ are due to the inability of PLL to form a complete helix in aqueos solution or whether these values are characteristic of PLL. Cassim and Taylor ${ }^{15}$ suggested from the ORD measurements that poly(L-glutamic acid) might not be able to form complete helixes in aqueous solution. In our results, almost all $\mathrm{CD}$ spectra of the mixtures show the pattern typical of an $\alpha$-helix. The relatively high $-[\theta]_{222}$ values of the mixtures may be understandable if we assume that the remaining non- $\alpha$-helical parts of PLL take the $\alpha$-helical conformation because of the existence of PAA.

\section{Effect of Molecular Weight of PAA}

In order to examine the effect of the degree of polymerization $\overline{\mathrm{DP}}$ on the interaction, PAA with relatively low molecular weight $(\overline{\mathrm{DP}}=80)$ (PAA-L) was used. As shown in Figures 1, 2, 


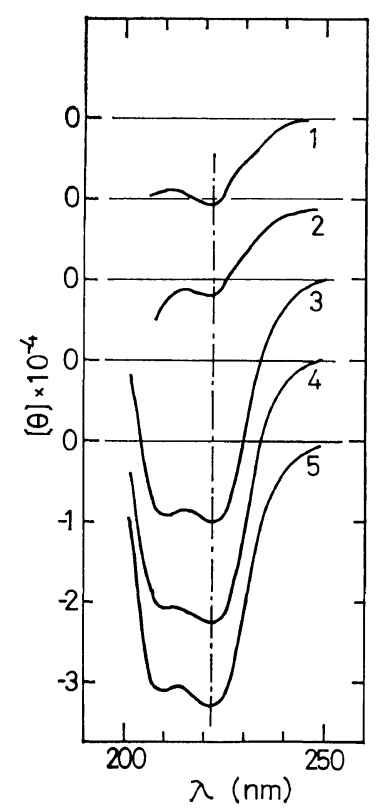

Figure 7. $\mathrm{CD}$ spectra of PLL-PAA-L mixtures at $25^{\circ} \mathrm{C}$ and various $\mathrm{pH}$ values. The mole ratios of PLL to PAA-L and the pH's are: (1) $67: 33$, $\mathrm{pH}=4.80$; (2) $50: 50, \mathrm{pH}=4.30$; (3) $50: 50, \mathrm{pH}=$ 12.10 ; (4) $12: 88, \mathrm{pH}=6.15$; (5) $12: 88, \mathrm{pH}=12.20$.

and 7, hardly any difference in the $C D$ spectra was found between PLL-PAA-L and PLLPAA-H systems. Moreover, the numerial values of $-[\theta]_{222}$ of the PLL-PAA-L mixtures also coincide with those of the corresponding PLLPAA-H mixtures (see Figures 5 and 7). This result shows that the effect of the molecular weight of PAA on the interaction with PLL is negligible in this $\overline{\mathrm{DP}}$ region $(80-3,100)$.

\section{Structure of Complex}

The CD spectra of the mixture in the UV region stated above only give information about the conformations of the PLL in the complex. In order to examine the conformation of PAA in the complex, the technique of induced circular dichroism may be useful. PAA is not optically active under ordinary conditions. Acridine orange, a symmetrical basic dye which often is used to analyze the conformations of acidic biopolymers, could bind to PAA, and may give information about the conformation of PAA. Figure 8 shows a CD spectrum of a PLL-PAA-H$\mathrm{AO}$ system at $\mathrm{pH}$ 7.11. The induced $\mathrm{CD}$ spectrum

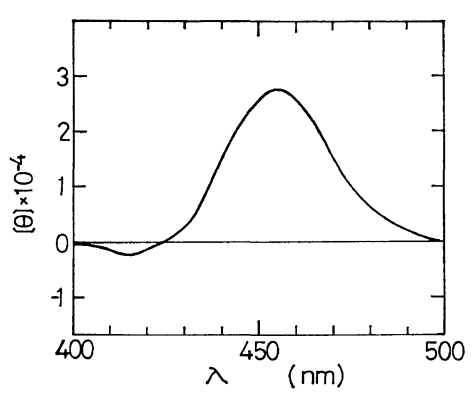

Figure 8. $\mathrm{CD}$ spectrum of PLL-PAA-H-AO system at $\mathrm{pH}$ 7.11. Molar ellipticity is calculated based on the molar concentration of $\mathrm{AO}$ : [PLL]= $0.0027 N ;$ [PAA-H] $=0.0028 N ;[$ AO $]=0.00030 N$.

has a large positive peak at $455 \mathrm{~nm}$ and a small negative peak at $415 \mathrm{~nm}$, and may indicate that the PAA forms a left-handed super helix. Taking into consideration the results of the poly(L-glutamic acid) and AO systems, ${ }^{16,17}$ the bands at 455 and $415 \mathrm{~nm}$ may be attributed to the electronic transitions polarized parallel and perpendicular to the axis of the helix. Bound AO may influence the conformation of the PAA. Hasumi, et $a l .{ }^{18}$ indicated in their study of the interaction on PLL-DNA-9-aminoacridine system that the interactions between PLL and DNA are much stronger than those between AO and DNA. In the PLL-PAA-AO system, AO would be expected to bind the carboxylic groups in the complex, but would not have a decisive influence on the structure of the complex. We concluded that PAA is able to form a left-handed super helix bound to the core composed of a right-handed $\alpha$-helix of PLL in the complex. This structure may be mainly due to the flexibility of the backbone chain of PAA.

The present study has shown that PAA interacts with PLL stoichiometrically and induces the formation of $\alpha$-helix of PLL, and further that the PAA may twist around the PLL helix.

\section{REFERENCES}

1. J. M. Rifkind and G. L. Eichhorn, Biochemistry 9, 1753 (1970).

2. A. Roychoudhyry, B. B. Biswas, and M. K. Pal, Makromol. Chem., 124, 113 (1969).

3. R. A. Gelman and J. Blackwell, Biopolymers 13, 139 (1974). 
4. A. Nakajima, K. Shinoda, T. Hayashi, and H. Sato, Polymer J., 7, 550 (1975).

5. W. B. Gratzer and P. McPhie, Biopolymers 4, 601 (1966).

6. A. B. Zezin, V. V. Lutsenko, V. B. Rogacheva, O. A. Aleksina, R. I. Kalyuzhnaya, V. A. Kabanov, and V. A. Kargin, Vysokomol. Soedin., A14(4), 772 (1972).

7. B. C. Myhr and J. G. Foss, Biopolymers 10, 425 (1971).

8. K. Shinada, T. Hayashi, and A. Nakajima, Polymer J., to be submitted.

9. G. Holzwarth and P. Doty, J. Amer. Chem. Soc. 87, 218 (1965).

10. A. Takahashi and M. Nagasawa, ibid., 86, 543 (1964).

11. C. Ciferri, D. Puett, L. Rajagh, and J. Hermans
Jr., Biopolymers 6, 1019 (1968).

12. M. L. Tiffany and S. Krimm, ibid., 12, 575 (1973).

13. M. Hatano and M. Yoneyama, J. Amer. Chem. Soc. 92, 1392 (1970).

14. R. A. Gelmam, W. B. Rippon, and J. Blackwell, Biopolymers 12, 541 (1973).

15. J. Y. Cassim and E. W. Taylor, Biophys. J. 5, 573 (1965).

16. R. E. Ballard, A. J. McCaffery, and S. F. Mason, Biopolymers 4, 97 (1966).

17. M. Hatano, M. Yoneyama, and Y. Sato, ibid., 12, 895 (1973).

18. H. Hasumi, K. Akasaka, H. Hatano, and K. Hiromi, Biochem. Biophys. Res. Commun. 50, 992 (1973). 